\title{
Metal-Organic Frameworks as Platforms for the Nanostructuration of Single Molecule Magnets: New Insights from HRTEM
}

\author{
Juby Varghese ${ }^{1}$, Darpandeep Aulakh ${ }^{1}$, Kyle Duell ${ }^{1}$, Timur Islamoglu ${ }^{2}$, \\ Haomiao $\mathrm{Xie}^{3}$, Lingmei Liu ${ }^{4}$, Omar Farha ${ }^{2}$, Kim R. Dunbar ${ }^{3}$, Yu Han ${ }^{4}$, \\ and Mario Wriedt ${ }^{1}$ \\ ${ }^{1}$ Department of Chemistry \& Biomolecular Science, Clarkson University \\ ${ }^{2}$ Department of Chemistry, Northwestern University \\ ${ }^{3}$ Department of Chemistry, Texas A\&M University \\ ${ }^{4}$ Advanced Membranes and Porous Materials Center, King Abdullah \\ University of Science and Technology \\ varghejr@clarkson.edu
}

Next-generation computer technologies require ultrahigh-density data storage devices and quantum computing based on isolated spin-carriers, so-called molecular spintronics. Singlemolecule magnets (SMMs) show great potential for such applications due to their unique magnetic properties. Their magnetic bistability allows switching from total spin up to total spin down on a molecular level where each molecule serves as a magnetic bit of information. The quest for the design of new SMMs with improved properties is ongoing, however, coupling of the nanoscale units to the macroscopic world remains a major challenge. Practical applications of SMMs require their organization in 2D or 3D networks while being in a protected environment to enable read-and-write processes. Metal-organic frameworks (MOFs), due to their porous nature and tunability, are excellent candidates to overcome this challenge. This work is the first systematic investigation exploring MOFs as platforms for the controlled nanostructuration of molecular magnets in both bulk polycrystalline powder and thin film forms. Subsequent characterization of SMM@MOF composites using HRTEM provides previously inaccessible visual structural insights into these nanomagnetic composites bringing us one step closer to the development of spintronic devices combining functional properties of both SMMs and MOFs.

Key words: Porous Frameworks, MOFs, Nanostructuration, SMMs, Data Storage, HRTEM 